\title{
A secretory hexokinase plays an active role in the proliferation of Nosema bombycis
}

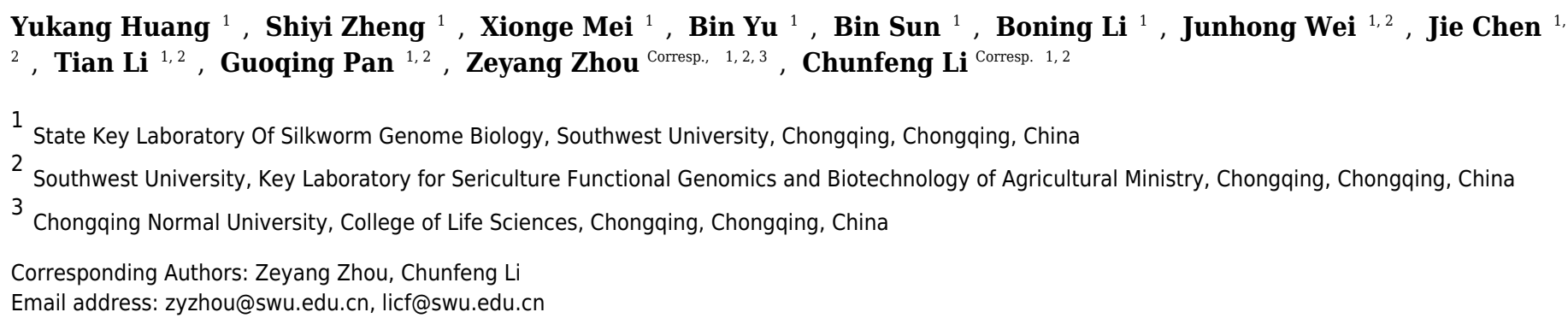

The microsporidian Nosema bombycis is an obligate intracellular parasite of Bombyx mori, that lost its intact tricarboxylic acid cycle and mitochondria during evolution but retained its intact glycolysis pathway. $N$. bombycis hexokinase ( $\mathrm{NbHK}$ ) is not only a rate-limiting enzyme of glycolysis but also a secretory protein. Indirect immunofluorescence assays and recombinant HK overexpressed in BmN cells showed that NbHK localized in the nucleus and cytoplasm of host cell during the meront stage. When $N$. bombycis matured, NbHK tended to concentrate at the nuclei of host cells. Furthermore, the transcriptional profile of $\mathrm{NbHK}$ implied it functioned during $\mathrm{N}$. bombycis' proliferation stages. A knock-down of $\mathrm{NbHK}$ effectively suppressed the proliferation of $N$. bombycis indicating that $\mathrm{NbHK}$ is an important protein for parasite to control its host. 


\section{A secretory hexokinase plays an active role in the}

\section{2 proliferation of Nosema bombycis}

3 Yukang Huang ${ }^{1}$, Shiyi Zheng ${ }^{1}$, Xionge $\mathrm{Mei}^{1}$, Bin $\mathrm{Yu}^{1}$, Bin Sun ${ }^{1}$, Boning $\mathrm{Li}^{1}$, Junhong Wei ${ }^{1,2}$, Jie

4 Chen ${ }^{1,2}$, Tian $\mathrm{Li}^{1,2}$, Guoqing Pan ${ }^{1,2}$, Zeyang Zhou ${ }^{1,2,3},{ }^{*}$ Chunfeng $\mathrm{Li}^{1,2, *}$

$5 \quad{ }^{1}$ State Key Laboratory of Silkworm Genome Biology, Southwest University, Chongqing, P. R.

6 China

$7{ }^{2}$ Key Laboratory for Sericulture Functional Genomics and Biotechnology of Agricultural

8 Ministry, Southwest University, Chongqing, P. R. China

$9 \quad{ }^{3}$ College of Life Sciences, Chongqing Normal University, Chongqing, P. R. China

11 Corresponding authors:

12 Zeyang Zhou ${ }^{1,2,3}$

13 Tiansheng Street, Chongqing, 400716, P. R. China

14 zyzhou@swu.edu.cn (ZeYang Zhou)

15 Chunfeng $\mathrm{Li}^{1,2}$

16 Tiansheng Street, Chongqing, 400716, P. R. China

17 licf@swu.edu.cn (Chunfeng Li) 


\section{Abstract}

The microsporidian Nosema bombycis is an obligate intracellular parasite of Bombyx mori, that lost its intact tricarboxylic acid cycle and mitochondria during evolution but retained its intact glycolysis pathway. $N$. bombycis hexokinase $(\mathrm{NbHK})$ is not only a rate-limiting enzyme of glycolysis but also a secretory protein. Indirect immunofluorescence assays and recombinant HK overexpressed in BmN cells showed that $\mathrm{NbHK}$ localized in the nucleus and cytoplasm of host cell during the meront stage. When $N$. bombycis matured, $\mathrm{NbHK}$ tended to concentrate at the nuclei of host cells. Furthermore, the transcriptional profile of $\mathrm{NbHK}$ implied it functioned during $N$. bombycis' proliferation stages. A knock-down of $N b H K$ effectively suppressed the proliferation of $N$. bombycis indicating that $\mathrm{NbHK}$ is an important protein for parasite to control its host.

\section{Introduction}

Microsporidia are unicellular eukaryotes and obligate intracellular parasites that have broad host range (Cali \& Takvorian 1999). Microsporidia can infect invertebrates and vertebrates including human beings (Cali \& Takvorian 1999; Franzen 2004). The life cycle of a microsporidia can be divided into the dormant extracellular and intracellular proliferation stages (Bigliardi \& Sacchi 2001). As obligate parasites, most microsporidia no longer have an intact tricarboxylic acid cycle or mitochondria, which implies an acute dependence on host energy during the intracellular stage (Hacker et al. 2014). In Encephalitozoon cuniculi and Trachipleistophora hominis, a series of nucleotide transport proteins (NTTs) can steal ATP from hosts (Heinz et al. 2014; Tsaousis et al. 2008). Owing to the absence of oxidative phosphorylation in microsporidia, glucose metabolism by glycolysis releases 7\% of full ATP potential (Berg et al. 2007). Even so, some microsporidia still maintain their intact glycolytic pathways, which may function during the extracellular stage (Wiredu et al. 2017). Although some microsporidia, such as Enterocytozoon bieneusi, lack its intact glycolytic pathway, most microsporidia still possess a hexokinase (HK) (Wiredu et al. 2017), which implies that HK may be a necessary protein for the infection and proliferation of microsporidia. 
Hexokinase is a multifunctional protein that can act in transcription regulation (Herrero et al. 1995; Niederacher \& Entian 1991) and apoptosis (Bryson et al. 2002; Gottlob et al. 2001), as well as with the cytokine neuroleukin (a nerve growth factor) (Chaput et al. 1988; Faik et al. 1988; Gurney et al. 1986). Furthermore, HK also participates in interaction between pathogenhost. For example, in macrophage, HK is an innate immune receptor for the detection of bacterial peptidoglycan (Wolf et al. 2016). In intracellular pathogen, such as Plasmodium falciparum, HK usually plays a basic role in the conversion of glucose to glucose 6-phosphate (Faik et al. 1988). A pathogen secreted HK was first reported in the microsporidia Nematocida parisii (Cuomo et al. 2012),which was also demonstrated using biochemical experiments (Reinke et al. 2017). This phenomenon was reported in Paranosema (Antonospora) locustae as well. The P. locustae HK was predicted to contain a signal peptide, and the indirect immunofluorescent assay (IFA) showed a nuclear localization in the host (Senderskiy et al. 2014; Timofeev et al. 2017). Then, special secreting hexokinase function which work as a regulator to increase ATP generation to parasite surface, was explored in Trachipleistophora hominis (Ferguson \& Lucocq 2018).

Nosema bombycis can infect Lepidoptera, including the economical insect silkworm, causing pebrine disease by vertical and horizontal transmission (Han \& Watanabe 1988). During infection, $N$. bombycis synthesizes many kinds of secretory proteins to control the host cells (Tian 2013). Just like P. locustae, N. bombycis has an intact glycolysis pathway (Wiredu et al. 2017). Therefore, we hypothesized that NbHK can be secreted into host cells and has a significant impact on N. bombycis' proliferation.

\section{Materials \& Methods}

\section{Hexokinase sequence analysis and ORF amplification}

4 The amino acid sequence of $\mathrm{NbHK}$ was submitted to SignalP 4.1 Server 5 (http://www.cbs.dtu.dk/services/SignalP/) and NCBI (https://www.ncbi.nlm.nih.gov/) for signal peptide and domain predictions. The HK of N. bombycis (GenBank Accession No. EOB11276.1) 
was amplified from genomic DNA (gDNA) by PCR. The amplification reaction consisted of 30 cycles of $94{ }^{\circ} \mathrm{C}$ for $15 \mathrm{~s}, 55{ }^{\circ} \mathrm{C}$ for $30 \mathrm{~s}$, and $72{ }^{\circ} \mathrm{C}$ for 1 min using the forward primer 5 'CGCGGATCCATGATAATTTTCTATTGT-3', containing a BamHI restriction site (GGATCC), and the reverse primer 5'-CCGCTCGAGATAAATAATTCGATGTAAAG-3' containing a XhoI restriction site (CTCGAG). PCR products were recovered (Omega, Norcross, GA, USA), integrated into pET-32 vector (TaKaRa, Japan), then the recombinant vectors were transformed into Escherichia coli DH5 $\alpha$ competent cells. The identified pET-32a-NbHK vector was sequenced by Sangon (Shanghai, China).

\section{Protein expression, purification and polyclonal antibody preparation}

The pET-32a- $N b H K$ vector was transformed into $E$. coli Rosetta for expression. After cultivation in $37{ }^{\circ} \mathrm{C}$, the recombinant bacteria was induced for $20 \mathrm{~h}$ at $16{ }^{\circ} \mathrm{C}$ with $1 \mathrm{mM}$ IPTG in LB medium. Nickel chelating affinity chromatography (Roche, Switzerland) was used to purify the target protein fused with hexahistine. BALB/c mice was used to generate antiserum by immunizing with $100 \mu \mathrm{g}$ recombinant $\mathrm{HK}$ protein homogenized with Freund's adjuvant $(\mathrm{V} / \mathrm{V}=1: 1$, Sigma-Aldrich) four times. Complete Freund's adjuvant was used in the first injection, then the incomplete Freund's adjuvant was used in the following injections. All animal experiments were approved by Laboratory Animals Ethics Review Committee of Southwest University guidelines (Chongqing, China) (Permit Number: SYXK- 2017-0019).

\section{Indirect immunoinfluscent assay}

Infected cells and healthy cells were fixed in PBS with 4\% paraformaldehyde, then treated with $0.5 \%$ Triton $\mathrm{X}-100$ for $10 \mathrm{~min}$ at room temperature, blocked with blocking regent $[0.5 \%(\mathrm{v} / \mathrm{v})$ bovine serum albumin and 10\%(w/v) goat serum] for $1 \mathrm{~h}$, incubated with 1:500 dilutions of HKantiserum (mouse) and $\mathrm{Nb} \beta$-tubulin polyclonal antibody (rabbit) at $37{ }^{\circ} \mathrm{C}$ for $60 \mathrm{~min}$. After washing with PBS three times (5 min each time), Alexa Fluor 488 and 594 (Thermo Fisher, CA, USA) were used to detect the binding of primary antibodies at room temperature. The nuclei were stained with 4',6-diamidino-2-phenylindole (DAPI) (Thermo Fisher, CA, USA) for 10 min. 
103 Samples were observed and imaged with Olympus FV1200 (Olympus, Japan).

104 Protein preparation

105 Protein of infected cells, mature spores and healthy cells were prepared by the glass-bead

106 breaking method as report (Geng et al. 2013). The samples with 0.4 g glass beads $(212-300 \mu \mathrm{m})$,

107 were lysed in RIPA Lysis Buffer (Beyotime, China) containing a protease inhibitor 108 (phenylmethylsulfonyl fluoride), and then crushed for 5 min at $4{ }^{\circ} \mathrm{C}$ using a Bioprep-24 109 Homogenizer (ALLSHENG. China). The treated samples were centrifuged at 12, $000 \mathrm{rpm}$ for 5 $110 \mathrm{~min}$ and the supernatant were collected.

\section{Immunoblot analysis}

112 Proteins were separated by sodium dodecyl SDS-PAGE and transferred to polyvinylidene 113 fluoride (PVDF) membrane (Roche, Switzerland). After blocking in blocking buffer [5\% (w/v) 114 skim milk, $20 \mathrm{mM}$ Tris-HCl, $150 \mathrm{mM} \mathrm{NaCl}$ and 0.05\% Tween-20], membrane incubated with an 115 HK-antiserum or $\mathrm{Nb} \beta$-tubulin-antiserum (diluted 1:3000)(Chen et al. 2017), washed, and 116 incubated with HRP-labeled goat anti-mouse IgG (diluted 1:6,000; Sigma, Saint Louis, MI, 117 USA). The bound antibodies were detected by ECL Plus Western Blotting Detection Reagents 118 (Bio-Rad, Richmond, CA, USA). The protein concentrations were detected with BCA Kit 119 (Beyotime, China), and loading quantity of samples were normalized on the basis of $\mathrm{Nb} \beta$-tubulin 120 quantity.

\section{Expression of recombinant HK fused with DsRed in BmN}

$\mathrm{NbHK}$ was cloned using the forward primer 5'-GGGTACCATGATAATTTTCTATTGTCTAC3' or 5'- GGTACCTTAATTAAGACATTGGGAAATA-3' [to remove the signal peptide $(\Delta \mathrm{SG})$, each containing a KpnI restriction site (GGTACC) and the reverse primer 5'TGACCCTGAGCCTCCATAAATAATTCGATGTAAAG-3' containing a $\mathrm{G}_{3} \mathrm{~S}_{2}$ linker sequence (GGAGGCTCAGGGTCA). The DsRed, a red fluorescent protein, was cloned used the forward primers 5'-GGAGGCTCAGGGTCAATGGTGCGCTCCTCCAAGAAC-3' containing a $\mathrm{G}_{3} \mathrm{~S}_{2}$ 
128 linker sequence (GGAGGCTCAGGGTCA) and the reverse primer 5'129 CCTCGAGGCGGCCGCTACAGGAACAGG

$130-3$ ' containing a $X h o$ I restriction site (CTCGAG). Then the DsRed was linked to the $r H K$ and $131 r H K \Delta \mathrm{SG}$ respectively through a $\mathrm{G}_{3} \mathrm{~S}_{2}$ linker peptide by overlapping PCR. Then the overlapping 132 PCR products were integrated into pCR-Blunt II-TOPO vector (Thermo Fisher, CA, USA), after 133 which the products of linkage were transform into E. coli DH5 $\alpha$. The recombinant pCR-Blunt II134 TOPO vector contained the targets fragments, were extracted from E. coli DH5 $\alpha$. The above 135 vectors and pIZ/V5-His (Thermo Fisher, CA, USA) were digested by KpnI and XhoI (Thermo 136 Fisher, CA, USA). The digested $r H K$ and $r H K \Delta S G$ fusing with DsRed were integrated into 137 digested pIZ/V5-His, which was induced by T4 DNA ligase (New England Biolabs, MA, USA). $1382 \mu \mathrm{g}$ constructed vectors were transiently transfected into BmN cells using X-tremeGENE HP 139 DNA Transfecti Reagent (Thermo Fisher, CA, USA). Three days later, the transfected cells 140 nuclei were labeled with Hoechest (Thermo Fisher, CA, USA). Then the samples were examined 141 by confocal microscopy (Olympus, Japan).

142

143

144

145

146

147

148

149

150

151

152

153

\section{RNA interference (RNAi) fragments synthesis}

The sequence of $N b H K$ was submitted to BLOCK-iT ${ }^{\mathrm{TM}}$ RNAi Designer (http://rnaidesigner.thermofisher.com/rnaiexpress/design.do). A 352-bp fragment that contain five potential interferential dsRNA fragments was amplified by the primers F-RI-Hexokinase-T7 5'-TAATACGACTCACTATAGGGAGAAGGAATATACTTGTCTGGGA-3' and R-RIHexokinase-T7 5'-

TAATACGACTCACTATAGGGAGATTGACAGGTCTCTCAAATGC-3', each containing the T7 promoters (TAATACGACTCACTATAGGGAGA). The amplified product was used as template to synthesize dsRNA using a RiboMAX Large Scale System-T7 Kit (Promega, Madison, WI, USA). The dsRNA-EGFP, which was used as the mock group, was prepared with the same method by the primers F-RI-EGFP 5'TAATACGACTCACTATAGGGAGAACGGCAAGCTGACCCTGAA-3' and R-RI-EGFP 5'- 
154

155

156

157

158

159

160

161

162

163

164

165

166

167

168

169

170

171

172

173

174

175

176

177

TAATACGACTCACTATAGGGAGATGTTGTAGTTGTACTCCAG-3', each containing the T7 promoters as well.

\section{DNA and cDNA collection from infected samples}

Spores were separated from hemolymph of severely infected silkworm pupae. The spores which were pretreated with $0.1 \mathrm{~mol} / \mathrm{L} \mathrm{KOH}$, were added to the Sf9-III cells (cell: spores ratio, 1:5) (Kawarabata \& Ren 1984). Infected cells were collected at 1, 3, 5 day post infection (d. p. i) and stored in PBS or TRIzol (Invitrogen, CA, USA). The Newly molted $4^{\text {th }}$ instar silkworm larvae were oral fed with $1 \times 10^{7}$ spores per. Then, the infected silkworms' midguts were collected and stored in TRIzol at $-80^{\circ} \mathrm{C}$ immediately. The gDNA of infected Sf9-III cells was extracted using a DNA extraction Kit (Omega, Norcross, Georgia, USA), while the cDNA of infected cells and midguts were prepared using Total RNA Kit II (Omega, Norcross Georgia USA) and RT-PCR Kit (Promega, Madison, Wisconsin, USA). Samples were taken from three separate experimental, mock and blank groups at each time point.

\section{RNAi of $N$. bombycis in infected Sf9-III}

The Sf9-III (Thermo Fisher, CA, USA) cells were infected by using the previous method described above. After infection, $2 \mu \mathrm{g}$ dsRNAs of $N b H K$ or EGFP were transfected into Sf9-III respectively and then cultured in 6-wells plates (Saleh et al. 2016). The samples were collected at 1,3 and 5 d. p. i.

\section{Cell counting}

The cell samples were suspended with $1 \mathrm{~mL}$ culture medium, then $10 \mu \mathrm{L}$ cell suspensions were mixed with $10 \mu \mathrm{L}$ trypan blue. Living counting were finished with Countess II FL (Thermo Fisher, CA, USA).

\section{Real-time quantitative PCR analysis}

For transcription detections, $1 \mu \mathrm{g}$ RNA was used to conduct reverse transcript PCR. Then, the 
178 products were diluted ten times as templates. Quantitative PCR was amplified using Hexokinase$179 \mathrm{qF}$

180 5'-CAAAATGTGATtATtATGGGAGATG-3' 3 and 5'181 CGATGtAAAgtATAAAGGGCTGAT-3' primers, and reference gene primers SSU-qF 5'182 CTGGGGATAGTATGATCGCAAGA-3'and SSU-qR 5'- CACAGCATCCATTGGAAACG-3' 183 (Huang et al. 2018). Quantitative PCR was performed with following program: a pre184 denaturation of $95{ }^{\circ} \mathrm{C}$ for $2 \mathrm{~min}$, followed by 40 cycles at $95{ }^{\circ} \mathrm{C}$ for $10 \mathrm{~s}$ and $60{ }^{\circ} \mathrm{C}$ for $20 \mathrm{~s}$ 185 (LightCycle 96, Roche, Switzerland). Expression of SfHk was detected using the same method 186 with SfHK-qF 5'-TCACTTACATTCAAGATTTACCCAA-3' and SfHK-qR 5'187 CTACGCCAGAACAAGAAAAGC-3' primers, and reference gene primers SfGAPDH-qF 5'GGCTGGCGCTGAATACATCGTTGAGTCCAC-3' and SfGAPDH-qR 5'TTAGCAACGGGAACACGGAAAGCCATACCAG-3'.

For $N b \beta$-tubulin copy number detection, $1 \mu \mathrm{L}(\sim 200 \mathrm{ng} / \mu \mathrm{L})$ of the each gDNA samples extracted from the above infected cells was analyzed by qPCR. The $10 \mu \mathrm{L}$ reaction systems were

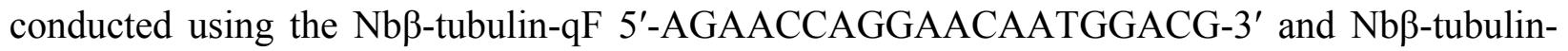
qR 5'-AGCCCAATTATTACCAGCACC-3' primers. Real-time PCRs were performed with the above program (LightCycle 96, Roche, Switzerland). The standard template had been constructed in previous research (Huang et al. 2018). The standard curve covered four orders of magnitude for the starting quantity $\left(1.3 \times 10^{3}-10^{6}\right)$. The multiple $\mathrm{T}$ tests were conducted with GraphPad.Prism.v6.01.

\section{Results}

\section{Recombinant hexokinase purification and immunoblot analysis}

The amino acid sequence analysis showed that $\mathrm{NbHK}$ contained a signal peptide, which implied it was a secretory protein (Fig $1 \mathrm{~A}$ ). The ORF of $H K$ was cloned by PCR using specific primers based on the gDNA of $N$. bombycis. The target gene were successfully integrated into pET-32a vector, which was validated by PCR and restriction enzyme digestion (Fig 1 B). The sequencing 
204

205

206

207

208

209

210

211

212

213

214

215

216

217

218

219

220

221

222

223

224

225

226

227

228

229

results showed a 1,287-bp fragment encoding 428 amino acids, which was consistent with the data from the genomic sequence of $N$. bombycis in SilkPathDB (https://silkpathdb.swu.edu.cn/)(Fig S1).

SDS-PAGE analysis showed that rHK was expressed at a molecular mass of $\sim 60 \mathrm{kDa}$, which was consistent with the predicted size (Fig $1 \mathrm{C}$ ). The purified protein was used to prepare the antibody. The titer of the HK antiserum was determined by ELISA (Fig S2). Western blot indicated that the $\mathrm{HK}$ antiserum can recognize a $50 \mathrm{kDa}$ protein specifically in the infected cells proteins, but the signal did not demonstrated in mature spores and healthy Sf9-III cells. (Fig 1 D). The molecular mass difference between rHK and native HK, which was consist in infected cells, was due to tag proteins.

\section{Subcellular localization of NbHK}

The subcellular localization of $\mathrm{NbHK}$ was analyzed by IFA. $\mathrm{Nb} \beta$-tubulin was used as a marker protein to distinguish the meront stage from mature spores. Meronts can be labeled using the $\mathrm{Nb} \beta$-tubulin antibody, while the mature spores can be observed visibly in differentialinterference microscopy (DIC) (Chen et al. 2017; Huang et al. 2018). The IFA demonstrated the $\mathrm{NbHK}$ can be secreted into host cells (Fig 2). In the proliferation stage, NbHK was located in the nucleus and cytoplasm of host cell (Fig 2 A-E). When N. bombycis matured, the NbHK tended to be concentrated in the nuclei of host cells (Fig 2 F-J). The change in localization implied that NbHK's functions might be different, depending on the growth stage of $N$. bombycis. The antibody of NbHK cannot bind to host cell in uninfected cells (Fig S3).

The vectors that encode fusion proteins HK::DsRed and HK $\Delta$ SG::DsRed were independently transfected into BmN cells (Fig 3 A). The fluorescence signal showed that rHK was localized in the cytoplasm of healthy cells, independent of presence of its signal peptide (Fig $3 \mathrm{~B}, \mathrm{C}$ ). When the BmN cells' nuclei became diffuse, indicating a trend toward apoptosis, the HK::DsRed colocalized with the nuclei (Fig $3 \mathrm{~B}$, white arrow). The similar results were demonstrated in transfected Sf9-III cells (Fig S4). 
230

231

232

233

234

235

236

237

238

239

240

241

242

243

244

245

246

247

248

249

250

251

252

253

\section{Transcriptional profile of $\mathrm{NbHK}$ in infected cells and midguts}

In infected Sf9-III cells, $N b H K$ was expressed at the early stage of infection and showed a highly significant increase from 8 to 48 hour post infection (h. p. i). Then, transcription levels were relatively stayed stable from 48 to 72 h.p.i and were slightly down-regulated from 72 to 96 h.p.i (Fig 4 A). In infected midgut of B.mori, the $N b H K$ expression pattern was similar to that of infected cells. $N b H K$ was gradually up-regulated, except for a slight down-regulation at 24 h. p. i (Fig $4 \mathrm{~B}$ ). The transcript profile of $N b H K$ implied it functioned during all proliferation stages of N. bombycis.

\section{Down-regulation of $\mathrm{NbHK}$ suppressed $\mathrm{N}$. bombycis' proliferation}

The effects of RNAi were assessed using qPCR and western blot. Transcriptional levels revealed that the expression of $\mathrm{NbHK}$ was significantly down-regulated by dsRNA in the experimental groups (dsRNA-HK) compared with the mock groups (dsRNA-EGFP) at 3 and 5 d.p.i (Fig 5 A). Besides, the western blot also showed $\mathrm{NbHK}$ down-regulated at $3 \mathrm{~d}$. p. i, which was basically consistent with transcriptional detection result (Fig 5 B).

The copy number of $N b \beta$-tubulin which lacks homology in the host was used to the reflect the infection level of the two groups (Huang et al. 2018). The infection of level the two groups kept similar at 1 d.p.i, because the effect of RNAi was not obvious at this time. In the mock groups, $N$. bombycis began to proliferate from 1 to 5 d.p.i while the proliferation of $N$. bombycis was remarkably inhibited in the experimental groups at 3 d.p.i (Fig 5 C).

The dsRNA-EGFP, dsRNA- $H K$ and transfection have no effect on proliferation of host cells and expression of SfHK (Fig S5 A, B). Besides, the RNAi constructs have no impact on proliferation of N. bombycis (Fig S6).

\section{Discussion}

$\mathrm{NbHK}$ was predicted to be a secretory protein with a signal peptide, suggesting its potential 
254 localization inside host cells. The location of $\mathrm{NbHK}$ during the proliferation stage of $N$. bombycis 255 infection implied it may play a role in regulating and controlling the host's energy metabolism 256 (Ferguson \& Lucocq 2018).. HK, with or without its signal peptide, and fused with DsRed was 257 expressed in $\mathrm{BmN}$ cells, which showed a similar subcellular localization. The localization of

258

259

260

261

262

263

264

265

266

267

268

269

270

271

272

273

274

275

276

277

278

279

280 eukaryotic expressed rHK revealed that the signal peptide could not lead the rHK to be secreted or to enter into nuclei of healthy $\mathrm{BmN}$ or Sf9-III. The localization results were different from those of $P$. locustae HK which is mainly concentrated on hosts' nuclei (Senderskiy et al. 2014; Timofeev et al. 2017). Expression profiles in vivo and in vitro also indicated $N b H K$ continuous high transcriptional level during proliferation stages of $N$. bombycis infection and the western blot showed $\mathrm{NbHK}$ barely exist in mature spores, which implied the NbHK just functioned in intracellular stage. But how microsporidia kept basic energy metabolism was still a secret. It is uncommon for an energy metabolism-related enzyme to be secreted into host cells and acted as a potential moderator. Although we showed that the down-regulation of $\mathrm{NbHK}$ expression can inhibit the proliferation of $N$. bombycis, the specific mechanisms involved need further study.

In microsporidia, there is only a tiny mitochondrial remnant called a mitosome (Goldberg et al. 2008; Williams et al. 2002), which implied that they are less prone to produce energy by themselves. The unique NTTs of microsporidia allow for a simplified energy metabolism pathway(Dean et al. 2018; Tsaousis et al. 2008). Although there is no ATP generation during the conversion of glucose to glucose 6-phosphate, it indirectly promotes glycolysis. The high transcription of $\mathrm{NbHK}$ and its importance function implied the glycolysis also play a key role during its intracellular stages, even microsporidia could steal ATP from host.HK is a control valve of glycolysis. The secretory HK of microsporidia turn up the valve of glycolysis and the tricarboxylic acid cycle, which is a clever strategy to steal energy from the host (Ferguson \& Lucocq 2018).

The lack of a stable and reliable gene manipulation method has impeded research on $N$. bombycis protein functions. In 2016, an EGFP was expressed in N. bombycis through a nontransposon vector (Rui et al. 2016). This was the first step toward the generation of successful 
281

282

283

284

285

286

287

288

289

290

291

292

293

294

295

296

297

298

299

300

301

302

303

304

305

306

307

transgenic microsporidia, but there were no follow-up reports regarding its application in protein function research. RNAi has been widely applied in other fungi including Nosema ceranae and Heterosporis saurida (Paldi et al. 2010; Saleh et al. 2016), to study protein functions. $N$. bombycis has all of the genes required for RNA silencing and it functions in vivo, but the RNA interference efficiency was unstable in different experimental groups (Pan et al. 2017; Wang et al. 2015). In our research, a stable RNAi strategy was established through an in vitro transcription system and lipofection in infected Sf9-III, which allowed high-throughput targets screening. Furthermore, RNAi in N. bombycis is not only a research method, but also an important strategy for breeding $N$. bombycis-resistant B. mori, which have been successful in BmNPV resistance (Kanginakudru et al. 2007).

\section{Conclusion}

The $N$. bombycis hexokinase is a secretory protein which localized in host cells' cytoplasm and nuclei. The $N b H K$ expressed during proliferation stage of infection in vivo and in vitro. The down-regulation of $\mathrm{NbHK}$ could inhibit the proliferation of this parasite. The results implied the NbHK was involved in controlling of host's energy metabolism.

\section{Acknowledgements}

This work was supported by the National Natural Science Foundation of China (Grant number: 31472151, 31702185) and Fundamental Research Funds for the Central Universities ((Grant number: XDJK2015A010, XDJK2018AA001).

\section{References}

Berg JM, Tymoczko JL, and Stryer L. 2007. Biochemistry: W. H. Freeman and Co.

Bigliardi E, and Sacchi L. 2001. Cell biology and invasion of the microsporidia. Microbes \& Infection 3:373-379.

Bryson JM, Coy PE, Gottlob K, Hay N, and Robey RB. 2002. Increased hexokinase activity, of either ectopic or endogenous origin, protects renal epithelial cells against acute oxidant-induced cell death. Journal of Biological Chemistry 277:11392-11400.

Cali A, and Takvorian PM. 1999. Developmental Morphology and Life Cycles of the Microsporidia: WileyBlackwell. 
Chaput M, Claes V, Portetelle D, Cludts I, Cravador A, Burny A, Gras H, and Tartar A. 1988. The neurotrophic factor neuroleukin is 90\% homologous with phosphohexose isomerase. Nature 332:454-455.

Chen J, Guo W, Dang X, Huang Y, Liu F, Meng X, An Y, Long M, Bao J, and Zhou Z. 2017. Easy labeling of proliferative phase and sporogonic phase of microsporidia Nosema bombycis in host cells. Plos One 12:e0179618.

Cuomo CA, Desjardins CA, Bakowski MA, Goldberg J, Ma AT, Becnel JJ, Didier ES, Fan L, Heiman DI, and Levin JZ. 2012. Microsporidian genome analysis reveals evolutionary strategies for obligate intracellular growth. Genome Research 22:2478-2488.

Dean P, Sendra KM, Williams TA, Watson AK, Major P, Nakjang S, Kozhevnikova E, Goldberg AV, Kunji ERS, and Hirt RP. 2018. Transporter gene acquisition and innovation in the evolution of Microsporidia intracellular parasites. Nature Communications 9.

Faik P, Walker JI, Redmill AA, and Morgan MJ. 1988. Mouse glucose-6-phosphate isomerase and neuroleukin have identical 3' sequences. Nature 332:455-457.

Ferguson S, and Lucocq J. 2018. The invasive cell coat at the microsporidian Trachipleistophora hominis-host cell interface contains secreted hexokinases. Microbiologyopen:e00696.

Franzen C. 2004. Microsporidia: how can they invade other cells? Trends in Parasitology 20:275-279.

Geng L, Wu H, Liu T, Tian R, Li T, Pan G, and Zhou Z. 2013. Two-dimensional Electrophoresis Analysis of Nosema bombycis Spore Coat Proteins Prepared by Germination Method and Glass Beads Breaking Method. Science of Sericulture 39:928-934.

Goldberg AV, Molik S, Tsaousis AD, Neumann K, Kuhnke G, Delbac F, Vivares CP, Hirt RP, Lill, Amp R, and Embley TM. 2008. Localization and functionality of microsporidian iron-sulphur cluster assembly proteins. Nature 452:624-628.

Gottlob K, Majewski N, Kennedy S, Kandel E, Robey RB, and Hay N. 2001. Inhibition of early apoptotic events by Akt/PKB is dependent on the first committed step of glycolysis and mitochondrial hexokinase. Genes Dev 15:1406-1418.

Gurney ME, Heinrich SP, Lee MR, and Yin HS. 1986. Molecular cloning and expression of neuroleukin, a neurotrophic factor for spinal and sensory neurons. Science 234:566-574.

Hacker C, Howell M, Bhella D, and Lucocq J. 2014. Strategies for maximizing ATP supply in the microsporidian Encephalitozoon cuniculi: direct binding of mitochondria to the parasitophorous vacuole and clustering of the mitochondrial porin VDAC. Cellular Microbiology 16:565-579.

Han MS, and Watanabe H. 1988. Transovarial transmission of two microsporidia in the silkworm, Bombyx mori, and disease occurrence in the progeny population. Journal of Invertebrate Pathology 51:41-45.

Heinz E, Hacker C, Dean P, Mifsud J, Goldberg AV, Williams TA, Nakjang S, Gregory A, Hirt RP, and Lucocq JM. 2014. Plasma membrane-located purine nucleotide transport proteins are key components for host exploitation by microsporidian intracellular parasites. Plos Pathogens 10:e1004547.

Herrero P, Galíndez J, Ruiz N, Martínez-Campa C, and Moreno F. 1995. Transcriptional regulation of the Saccharomyces cerevisiae HXK1, HXK2 and GLK1 genes. Yeast 11:137.

Huang Y, Chen J, Sun B, Zheng R, Li B, Li Z, Tan Y, Wei J, Pan G, and Li C. 2018. Engineered resistance to Nosema bombycis by in vitro expression of a single-chain antibody in Sf9-III cells. Plos One 13:e193065.

Kanginakudru S, Royer C, Edupalli SV, Jalabert A, Mauchamp B, Prasad SV, Chavancy G, Couble P, and Nagaraju J. 2007. Targeting ie-1 gene by RNAi induces baculoviral resistance in lepidopteran cell lines and in 
transgenic silkworms. Insect Molecular Biology 16:635-644.

Kawarabata T, and Ren I. 1984. Infection and development of Nosema bombycis (Microsporida: Protozoa) in a cell line of Antheraea eucalypti. Journal of Invertebrate Pathology 44:52-62.

Niederacher D, and Entian KD. 1991. Characterization of Hex2 protein, a negative regulatory element necessary for glucose repression in yeast. European Journal of Biochemistry 200:311-319.

Paldi N, Glick E, Oliva M, Zilberberg Y, Aubin L, Pettis J, Chen Y, and Evans JD. 2010. Effective gene silencing in a microsporidian parasite associated with honeybee (Apis mellifera) colony declines. Applied \& Environmental Microbiology 76:5960-5964.

Pan Q, Wang L, Dang X, Ma Z, Zhang X, Chen S, Zhou Z, and Xu J. 2017. Bacterium - Expressed dsRNA Downregulates Microsporidia Nosema bombycis Gene Expression. Journal of Eukaryotic Microbiology 64:278.

Reinke AW, Balla KM, Bennett EJ, and Troemel ER. 2017. Identification of microsporidia host-exposed proteins reveals a repertoire of rapidly evolving proteins. Nature Communications 8:14023.

Rui G, Cao G, Lu Y, Xue R, Kumar D, Hu X, and Gong C. 2016. Exogenous gene can be integrated into Nosema bombycis genome by mediating with a non-transposon vector. Parasitology Research 115:3093-3098.

Saleh M, Kumar G, Abdelbaki AA, Dkhil MA, Elmatbouli M, and Alquraishy S. 2016. In Vitro Gene Silencing of the Fish Microsporidian Heterosporis saurida by RNA Interference. Nucleic Acid Therapeutics 26:250-256.

Senderskiy IV, Timofeev SA, Seliverstova EV, Pavlova OA, and Dolgikh VV. 2014. Secretion of Antonospora (Paranosema) locustae proteins into infected cells suggests an active role of microsporidia in the control of host programs and metabolic processes. Plos One 9:e93585.

Tian LI. 2013. A Comparative Genomics Analysis on Secretory Proteins of Four Microsporidian Species. Science of Sericulture 39:527-536.

Timofeev SA, Senderskiy IV, Tsarev AA, Tokarev YS, and Dolgikh VV. 2017. Heterologous expression of Paranosema (Antonospora) locustae hexokinase in lepidopteran, Sf9, cells is followed by accumulation of the microsporidian protein in insect cell nuclei. Journal of Invertebrate Pathology 143:104-107.

Tsaousis AD, Kunji ER, Goldberg AV, Lucocq JM, Hirt RP, and Embley TM. 2008. A novel route for ATP acquisition by the remnant mitochondria of Encephalitozoon cuniculi. Nature 453:553-556.

Wang L, He C, Dang X, Xu J, and Zhou Z. 2015. Identification,Evolutionary and Transcriptional Activity Analyses of a Dicer-like Gene in Microsporidium Nosema bombycis. Science of Sericulture.

Williams BA, Hirt RP, Lucocq JM, and Embley TM. 2002. A mitochondrial remnant in the microcporidian Trachipleistophora hominis. Nature 418:865.

Wiredu BD, Jaroenlak P, Prachumwat A, Williams TA, Bateman KS, Itsathitphaisarn O, Sritunyalucksana K, Paszkiewicz KH, Moore KA, and Stentiford GD. 2017. Decay of the glycolytic pathway and adaptation to intranuclear parasitism within Enterocytozoonidae microsporidia. Environmental Microbiology 19.

Wolf AJ, Reyes CN, Liang W, Becker C, Shimada K, Wheeler ML, Cho HC, Popescu NI, Coggeshall KM, and Arditi M. 2016. Hexokinase Is an Innate Immune Receptor for the Detection of Bacterial Peptidoglycan. Cell 166:624-636. 


\section{Figure 1}

Expression and analysis of $\mathrm{NbHK}$.

(A) Protein structure of NbHK. There is a signal peptide contacting 23 amino acids in the $\mathrm{N}$ terminus. (B) Validation of the PET-32-NbHK vector by PCR (left) and Bam HI/Xho I enzyme digestion (right). Products $\sim 1,300$ bp were amplified by PCR or cleaved from the constructed vector. M: 2K Plus (TaKaRa, Japan). (C) Purification of recombinant HK. Recombinant HK was eluted by elution buffer containing different concentration of imidazole and analyzed by SDSPAGE. (D) Specificity of the HK antiserum. Proteins extracted from infected Sf9-III, mature spores and healthy Sf9-III were subjected to western blot using polyclonal antibody against HK. M: Protein maker (Transgene, China).

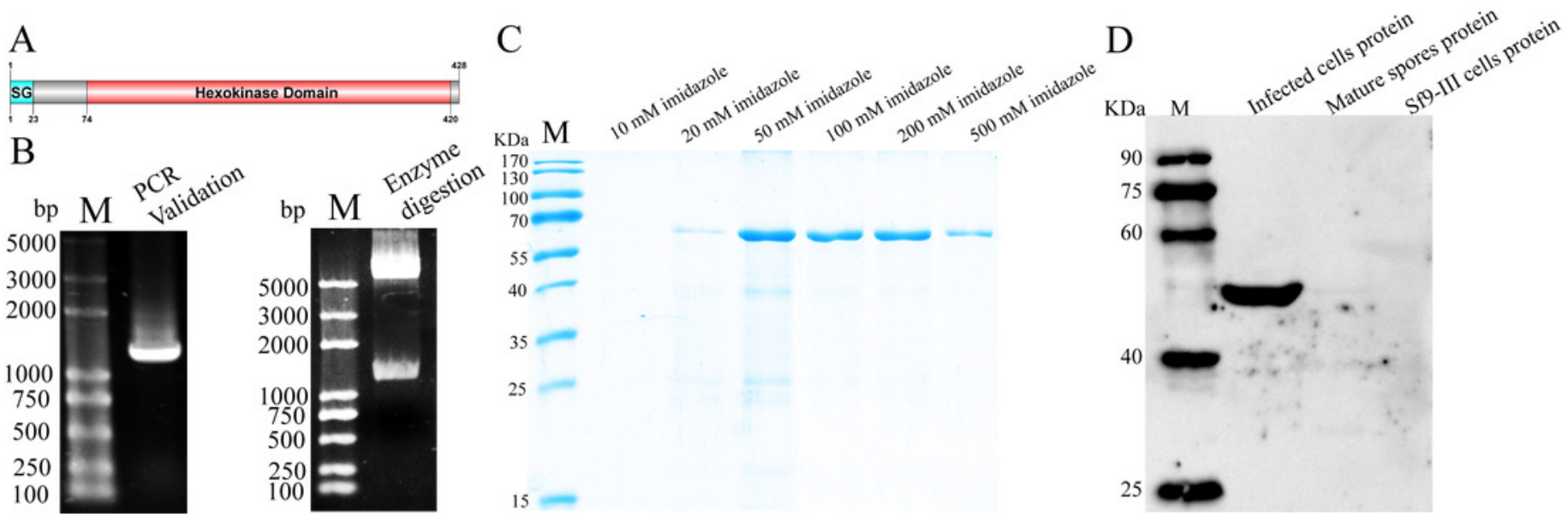




\section{Figure 2}

Subcellular localization of NbHK in infected Sf9-III.

Localization of NbHK was imaged with confocal microscopy at different stage of infection. Green and red fluorescence were observed in the samples incubated with the HK antibody (mouse) and rabbit $\mathrm{Nb \beta}$-tubulin antiserum, respectively. The nuclei of host cells and $N$. bombycis were labeled with DAPI ( blue fluorescent signal ) (Thermo Fisher, CA, USA). (A-E) Localization of $\mathrm{NbHK}$ in the meront stage of $\mathrm{N}$.bombycis. The parasites were bound by $\mathrm{Nb} \beta$ tubulin antibody, which showed that the meronts lacked a chitin layer. The NbHK dispersed in the whole host cell. (F-J) Localization of NbHK in the mature spore stage of N.bombycis. The chitin layer of the mature spore blocked the binding of the $\mathrm{Nb} \beta$-tubulin antibody, and the mature spore could be observed by DIC. NbHK was concentrated in host cell nuclei at this stage.

*Note: Auto Gamma Correction was used for the image. This only affects the reviewing manuscript. See original source image if needed for review.

DAPI
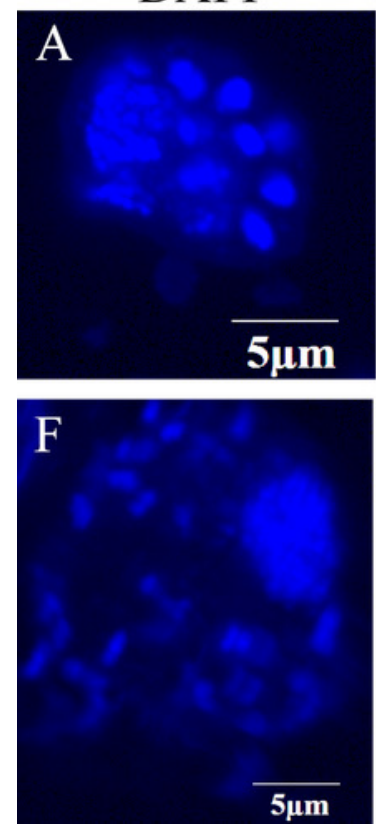

$\mathrm{NbHK}$
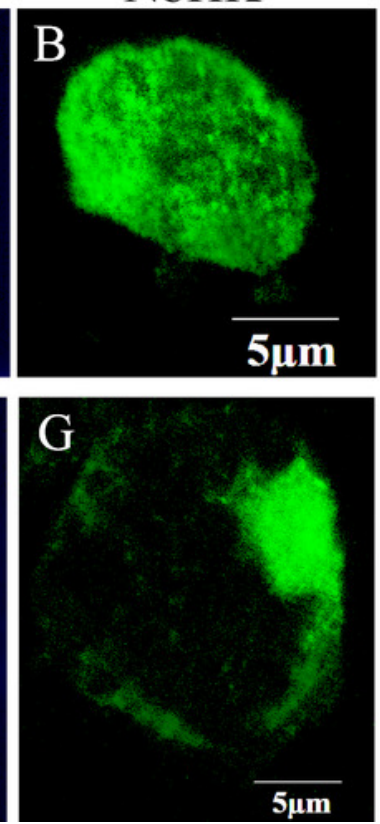

$\mathrm{Nb} \beta$-tubulin
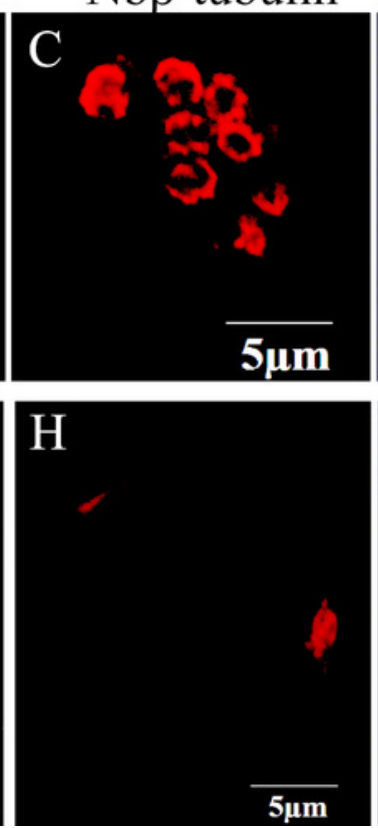

Merge
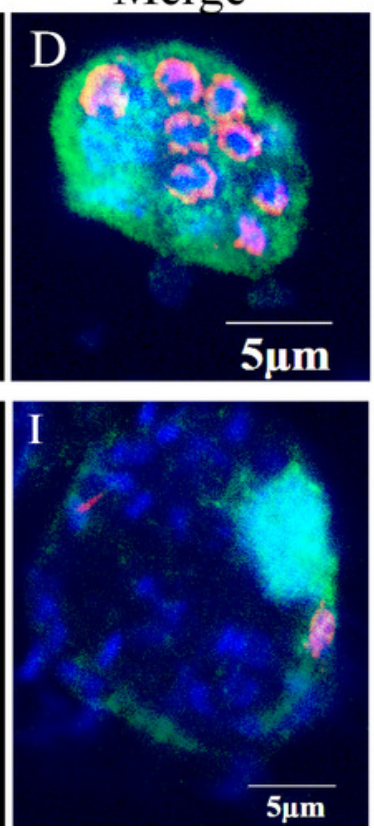

DIC
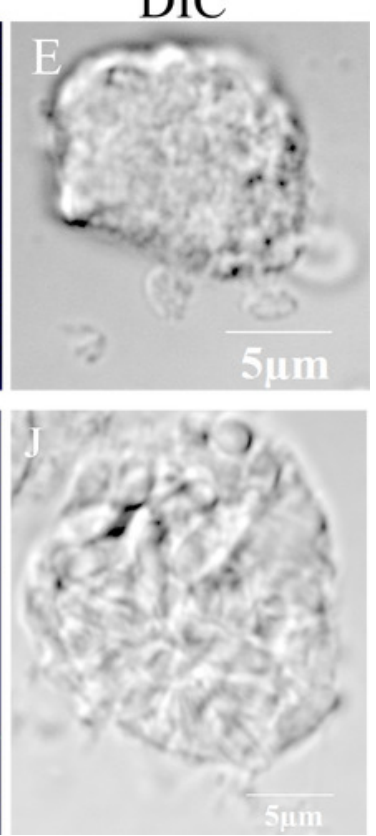


\section{Figure 3}

Subcellular localization of HK::DsRed and HKASG::DsRed in BmN.

(A) HK, with or without its signal peptide, was fused with DsRed. The two expression cassettes were integrated into plZ/v5-His, and then the constructed vectors were transfected into BmN. (B) Subcellular localization of HK::DsRed in BmN. The cell nucleus was labeled with Hoechest. The white arrow indicates an apoptotic cell. (C) Subcellular localization of HKASG::DsRed in BmN.

A

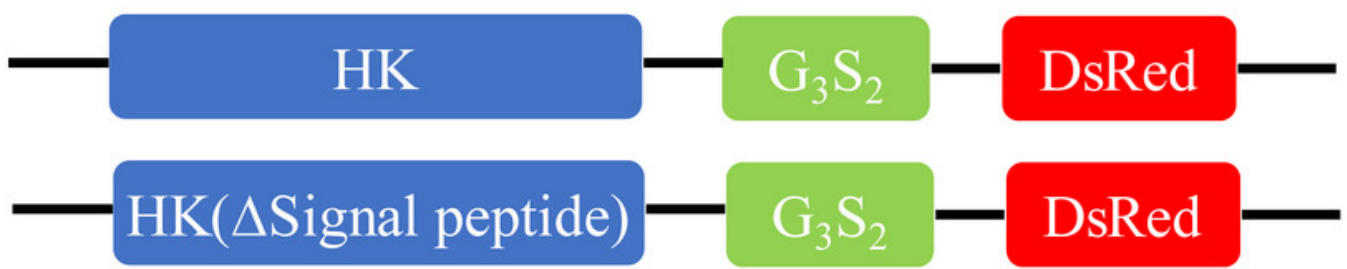

B
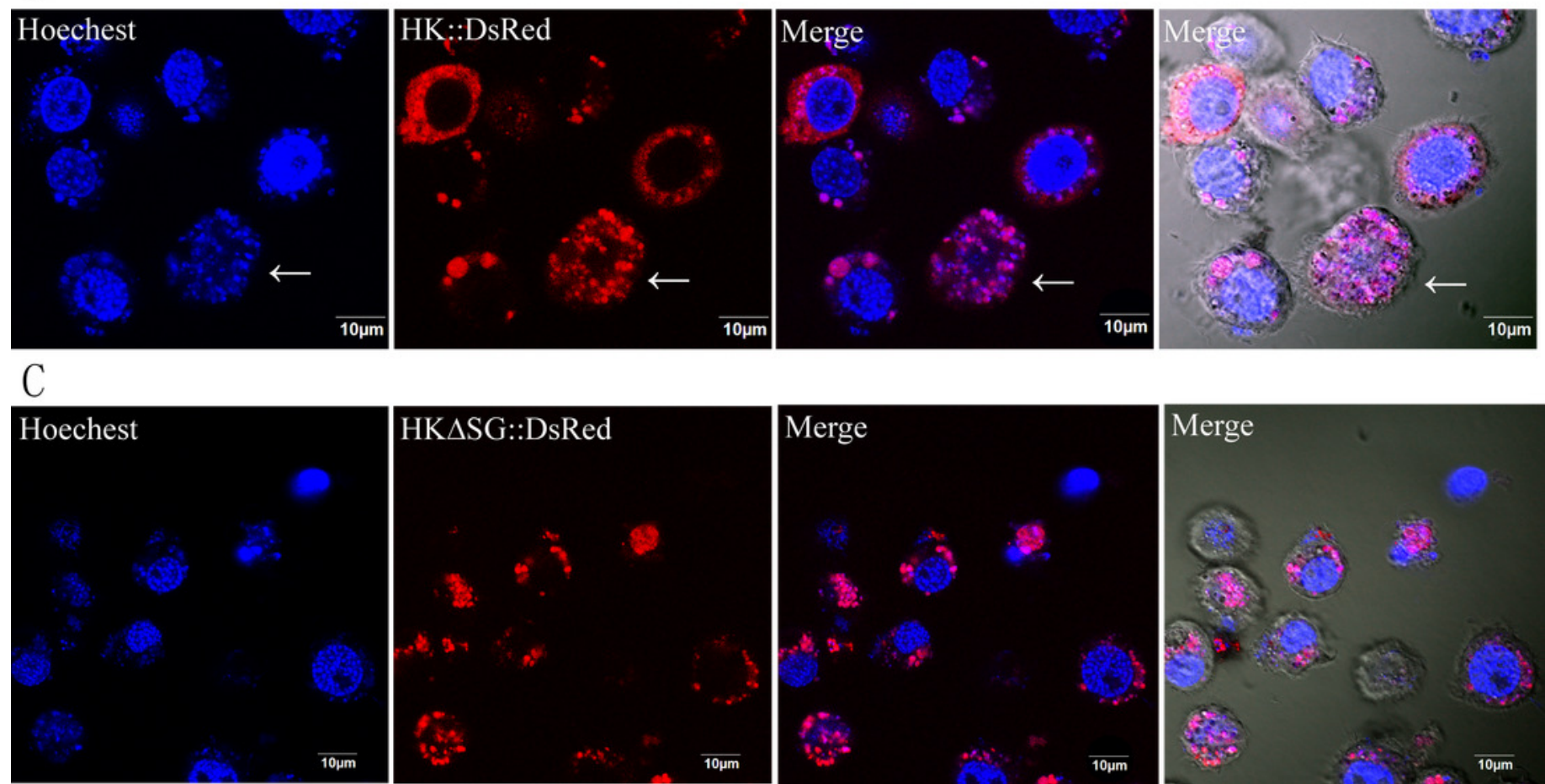


\section{Figure 4}

Transcriptional profile of NbHK in infected Sf9-III cells and midguts.

The relative expression level of $\mathrm{NbHK}$ in infected Sf9-III cells and midguts at different time points. They are presented relative to the 8 h.p.i. N. bombycis SSU rRNA was the reference gene for the normalization of expression levels. (A) Transcript level of NbHK in infected Sf9-III cells. (B) Transcript level of $N b H K$ in infected midgut of $B$. mori. Vertical bars show the mean \pm SEs $(n=3)$.
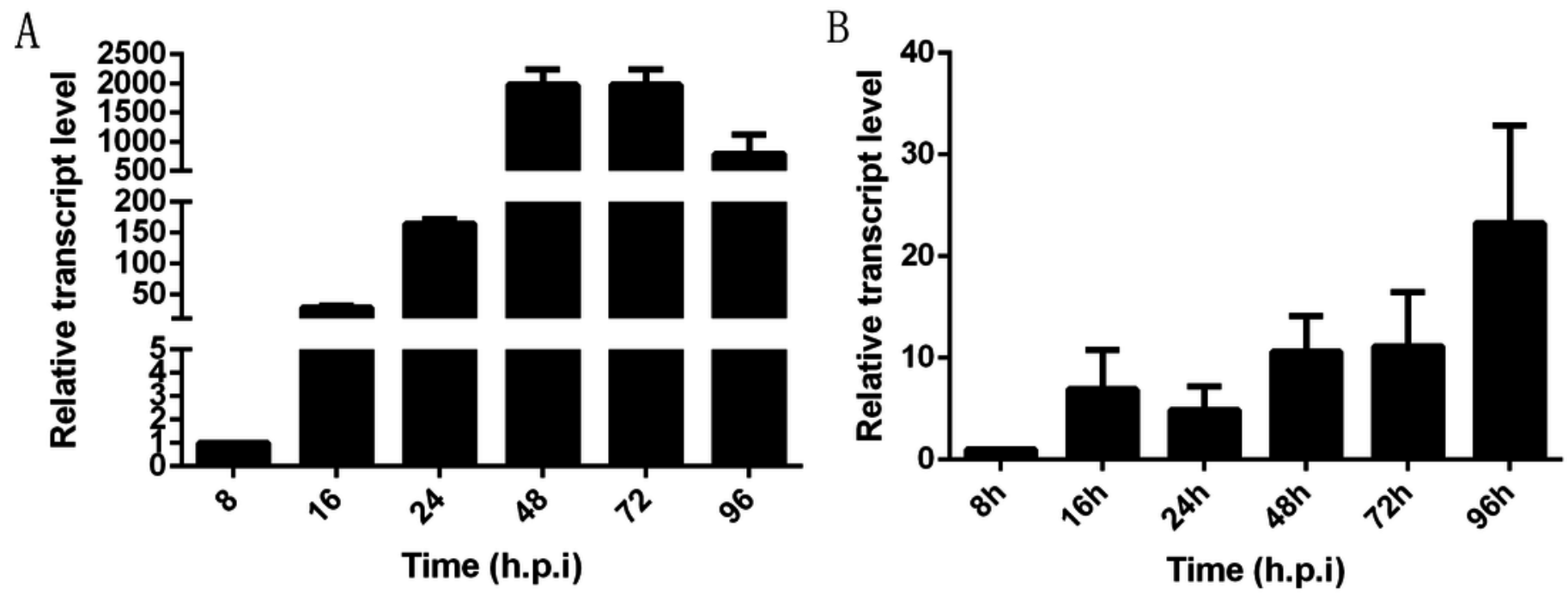


\section{Figure 5}

Effect of $\mathrm{NbHK}$ down-regulation on $\mathrm{N}$. bombycis proliferation.

(A) The transcription levels of NbHK. Complementary DNAs of the experimental (dsRNA-NbHK) and mock (dsRNA-EGFP) groups were analyzed by qPCR. The $\triangle \triangle \mathrm{C}_{\mathrm{t}}$ method was conduct to process the data. (B) NbHK expression levels of NbHK. Total proteins of experimental (dsRNA-NbHK) and mock (dsRNA-EGFP) groups were analyzed by western blot. Loading quantity of samples were normalized by $\mathrm{Nb} \beta$-tubulin, and $\mathrm{NbHK}$ quantities were detected with its antiserum. (C) Infection levels of Sf9-III. Genomic DNA was extracted from experimental (dsRNA-NbHK) and mock (dsRNA-EGFP) groups at 1, 3 and 5 d.p.i. Copy numbers of $\mathrm{Nb} \beta$-tubulin indicated the infection levels. Statistics significant differences are represented with asterisks $(* P<0.05 ; * * P<0.01)$. Bars represent the standard deviations of three independent replicates.
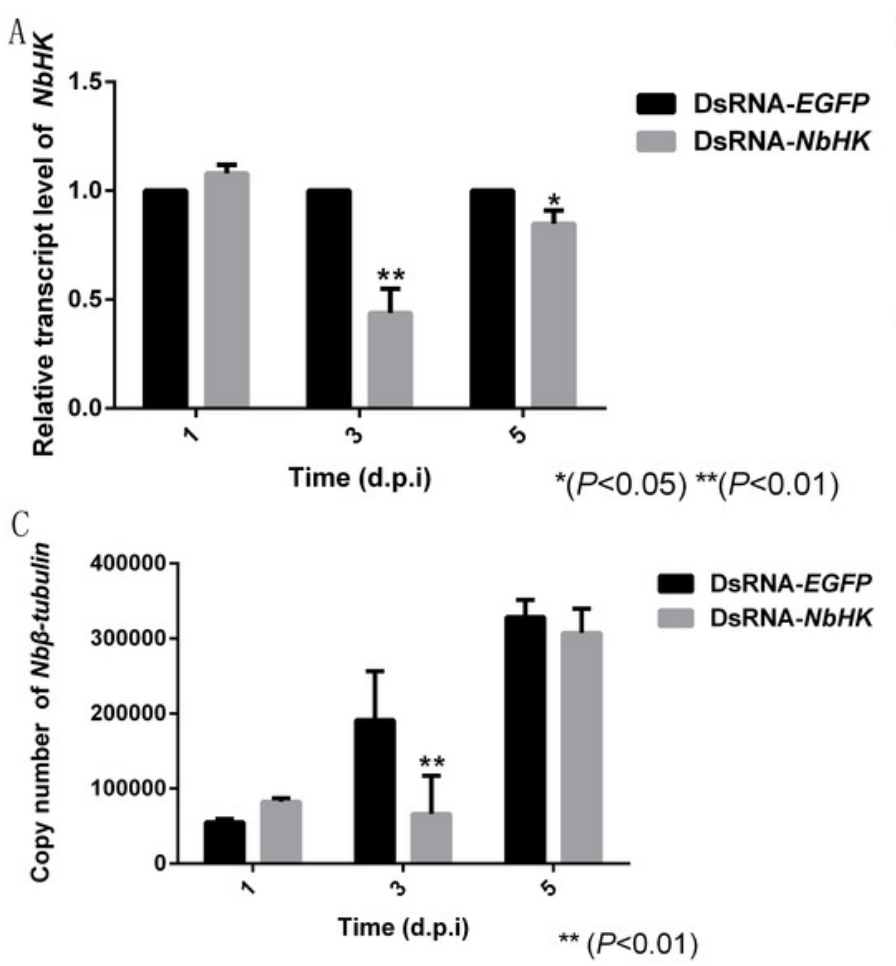

B

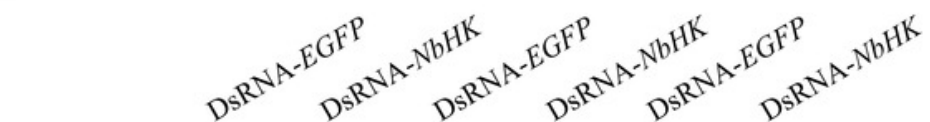

Nbhexokinase

$\mathrm{Nb} \beta$-tubulin

1 d.p.i

3 d.p.i

5 d.p.i 